\title{
Drought Risk Assessment of China's Mid-Season Paddy
}

\author{
Yongdeng Lei ${ }^{1,2}$, Jing'ai Wang ${ }^{1,2, *}$, and Lili Luo ${ }^{3,4}$ \\ ${ }^{1}$ School of Geography and Remote Sensing Science, Beijing Normal University, Beijing 100875, China \\ ${ }^{2}$ Key Laboratory of Regional Geography, Beijing Normal University, Beijing 100875, China \\ ${ }^{3}$ State Key Laboratory of Earth Surface Processes and Resource Ecology, Beijing Normal University, Beijing 100875, China \\ ${ }^{4}$ Hebi College of Vocation and Technology, Henan 458030, China
}

\begin{abstract}
China has the world's largest population and a large and critically important agricultural sector. Sixty-five percent of the Chinese population lives on paddy rice. However, drought disasters frequently afflict China's rural population and threaten its food security. It is therefore of paramount importance to assess the drought risk of paddy in China. We establish a quantitative risk assessment model for the drought risk of mid-season paddy and regional-specific vulnerability curves, evaluate the drought risk of mid-season paddy, and compile a series of risk maps. The drought disaster risk rating results indicate that risk is highest in Northeast China, followed by Northwest China, North China, and South China, showing a decreasing trend from north to south. The mid-season paddy area of Northeast China has the highest mean risk index (0.58-0.71), followed by northwestern provinces such as Inner Mongolia and Xinjiang (0.5-0.6), while risk indices in provinces of North China such as Hebei and Shandong range from $0.3-0.5$, and the southern provinces show a relatively low level of risk. This article presents the preliminary results of a scientific inquiry on where the high drought risk areas of mid-season paddy are and how high the risk is. These results provide a regional-specific basis for drought risk governance of paddy in China.
\end{abstract}

Keywords China, drought disaster risk, mid-season paddy, regional risk assessment, vulnerability curves

\section{Introduction}

Drought is the most widespread natural disaster and causes the biggest losses in agriculture around the world. Nearly half of the countries in the world suffer from drought (UNDP 2004; IPCC 2007). China experiences frequent, spatially extensive, and lasting droughts that cause the most severe losses to the agricultural economy (Wang et al. 2002; Wang et al. 2005). Paddy rice is the most important grain crop in China. Sixty-five percent of the Chinese population lives primarily on paddy rice and more than one third of the grain yield in China comes from rice paddy. In 2008, paddy rice

\footnotetext{
* Corresponding author. E-mail: sqq@bnu.edu.cn
}

constituted 37 percent of the total grain yield in China (Department of Cropping Administration, Ministry of Agriculture 2010). Drought disasters often cause reduction in rice production or even total crop failure. During the Chongqing summer drought in 2006, in Sichuan Province and Chongqing Municipality alone 3.2 million hectares of paddy rice and other autumn grain crops were affected with an unusually dry area of 1.87 million hectares and a production loss of 9.83 million tons, resulting in a direct economic loss of RMB 21.17 billion Yuan. According to available statistics, between 1978 and 2008, the annual average area of paddy suffering from drought in China reached 30 million hectares (NDRCC 2008). Drought disasters afflicting paddy rice production have severely threatened food security in China. Therefore, studying the drought risk of paddy in China and preventing catastrophic agricultural disaster risk have become urgent issues for the sustainable development of agriculture (Shi 2009).

Chinese paddy rice production is typically divided into early-season paddy, mid-season paddy (including singleseason paddy), and double-cropping late-season paddy. The proportions of total planted area in 2008 were 21 percent, 56 percent, and 23 percent, respectively. Since mid-season paddy constitutes the highest proportion of paddy yield and is most widespread, we studied the drought risk during its growing season. We established regional-specific drought vulnerability curves, conducted an assessment of drought risk nationwide, and analyzed the temporal-spatial patterns in order to provide a scientific basis for regional-specific drought disaster reduction of paddy. This is of great significance to reducing drought losses and stabilizing grain production in China.

\section{Basic Data}

According to the theory of regional disaster systems, drought disasters result from the combined effect of the instability of the disaster-formative environment, the severity of hazards, and the vulnerability of the exposure units (Shi 2002, 2005), paddy crops in this case. Therefore, the drought risk assessment 
of mid-season paddy needs to be based on historical data such as precipitation, exposure of paddy fields, and cases of drought losses in order to calculate vulnerability and the risk of drought disasters (Table 1).

To define the spatial exposure of mid-season paddy to drought, we extracted the polygon features labeled as paddy field on the 1:1,000,000 national land-use map by ArcGIS to approximate the distribution of Chinese mid-season paddy. By overlaying this result with China's paddy cropping regionalization map, we established a database of Chinese mid-season paddy (with a spatial resolution of $1 \mathrm{~km}$ ).

\section{Quantitative Risk Assessment Model for Drought Disaster of Mid-Season Paddy}

Usually, disaster risk is defined as the probability of a hazard in the future and the possible losses resulting from it: Disaster Risk $=\mathrm{f}$ (Hazard, Exposure, Vulnerability) (UNDP 2004; ADRC 2005). Since the distribution and cropping system of rice paddy in China are complex and natural conditions and drought vulnerability of paddy in different regions vary significantly, it is necessary to carry out regionalspecific assessments in the study of drought disaster risk of paddy. With regard to the reason for a regional-specific assessment (Why), the way to carry out this assessment (How), and the purpose of such an assessment (What), this research establishes a theoretical framework - the Regional Assessment-Vulnerability Variation (RAVV) framework (Figure 1).

Based on the RAVV framework, this research establishes a quantitative risk assessment model for drought disasters to calculate the exceeding probability of drought in the growing period of mid-season paddy and the regional-specific drought vulnerability curves for the four regions defined by the paddy cropping regionalization of China and calculate the risk probability distribution of drought disasters of different frequencies, and develops a $1 \mathrm{~km}$ grid risk map of mid-season paddy drought risk. This provides a quantitative basis for reducing drought vulnerability and the risk of drought disasters for paddy in China.

\subsection{Calculating Exceeding Probability of Drought Hazard Severity}

The major indicators for drought risk assessment include precipitation anomalies (in percent), the standard precipitation index (SPI), the relative moisture index (RMI), the compound drought index (CI), and the Palmer drought severity index (PDSI) (China Meteorological Administration 2006). Among them, precipitation anomalies can properly represent the abnormal conditions of precipitation within a certain period as compared to the average values. Because this indicator has a straightforward meaning and is simple to calculate, it is widely used. With the long-term seasonal (April-September) average precipitation at various weather stations from 1971-2000 as reference and daily precipitation for the same season from 1966-2005 (40 years) aggregated into seasonal totals for each year as input data, we calculate precipitation anomalies within the growing period of mid-season paddy (April-September) and use this as the main indicator for characterizing the severity of drought hazard (Eq. 1).

$$
h=\frac{P-\bar{P}}{\bar{P}} \times 100 \%
$$

Where, $h$ is drought hazard severity, representing the precipitation anomaly within a certain period of time, $P$ represents the actual precipitation $(\mathrm{mm})$ within the same time period, and $\bar{P}$ represents the long-term average precipitation $(\mathrm{mm})$ of the same period. The national standard Classification of Meteorological Droughts (GB/T20481-2006) issued by the China Meteorological Administration (2006) (Table 2) is used for categorizing drought hazard severity.

Exceeding probability (EP) of drought hazard is the probability that a certain magnitude of drought will be surpassed in a given time period, which can be calculated using Eq. 2. By calculating the exceeding probability at each weather station and through spatial interpolation in ArcGIS, we derive the spatial distribution of drought hazard severity $(h)$ for China at four recurrence intervals (once in 5 years, once in 10 years, once in 20 years, and once in 40 years) for each $1 \mathrm{~km} \times 1 \mathrm{~km}$ grid.

$$
P_{(h, t)}=1-(1-1 / t)^{n}
$$

Eq. 2

\begin{tabular}{|c|c|c|c|}
\hline Data & Description & Source & Year \\
\hline $\begin{array}{l}\text { Ground meteorological } \\
\text { observations }\end{array}$ & $\begin{array}{l}\text { Precipitation, temperature, and wind speed at } \\
752 \text { weather stations of China }\end{array}$ & $\begin{array}{l}\text { National Meteorological Information Center of } \\
\text { China Meteorological Administration }\end{array}$ & $1966-2005$ \\
\hline Land use & Spatial distribution of paddy fields & $\begin{array}{l}\text { 1:1,000,000 China Land-Use Vector Format GIS } \\
\text { Map (IGSNRR, CAS 2000) }\end{array}$ & 2000 \\
\hline Paddy-based production & Paddy yield and cropping area by counties & $\begin{array}{l}\text { China County and City Socioeconomic Statistical } \\
\text { Yearbook (National Bureau of Statistics of China 2001) }\end{array}$ & 2001 \\
\hline Paddy cropping regionalization & $\begin{array}{l}\text { China Paddy Cropping Regionalization Vector } \\
\text { Map }\end{array}$ & $\begin{array}{l}\text { Agridata (Chinese Academy of Agricultural Sciences } \\
\text { 2010) }\end{array}$ & 2010 \\
\hline Paddy phenology & China Paddy Phenological Period Vector Map & China National Agricultural Atlas (CAS 1989) & 1989 \\
\hline $\begin{array}{l}\text { Database of cases of drought } \\
\text { losses }\end{array}$ & $\begin{array}{l}\text { Drought-affected and severely impacted areas } \\
\text { and no harvest areas, production loss, etc. }\end{array}$ & $\begin{array}{l}\text { China Meteorological Disasters Collection (Ding 2008); } \\
\text { China Agricultural Statistical Data (Chen 2009) }\end{array}$ & $1970-2008$ \\
\hline
\end{tabular}

Table 1. Basic data sources for assessing the drought risk of mid-season paddy in China 


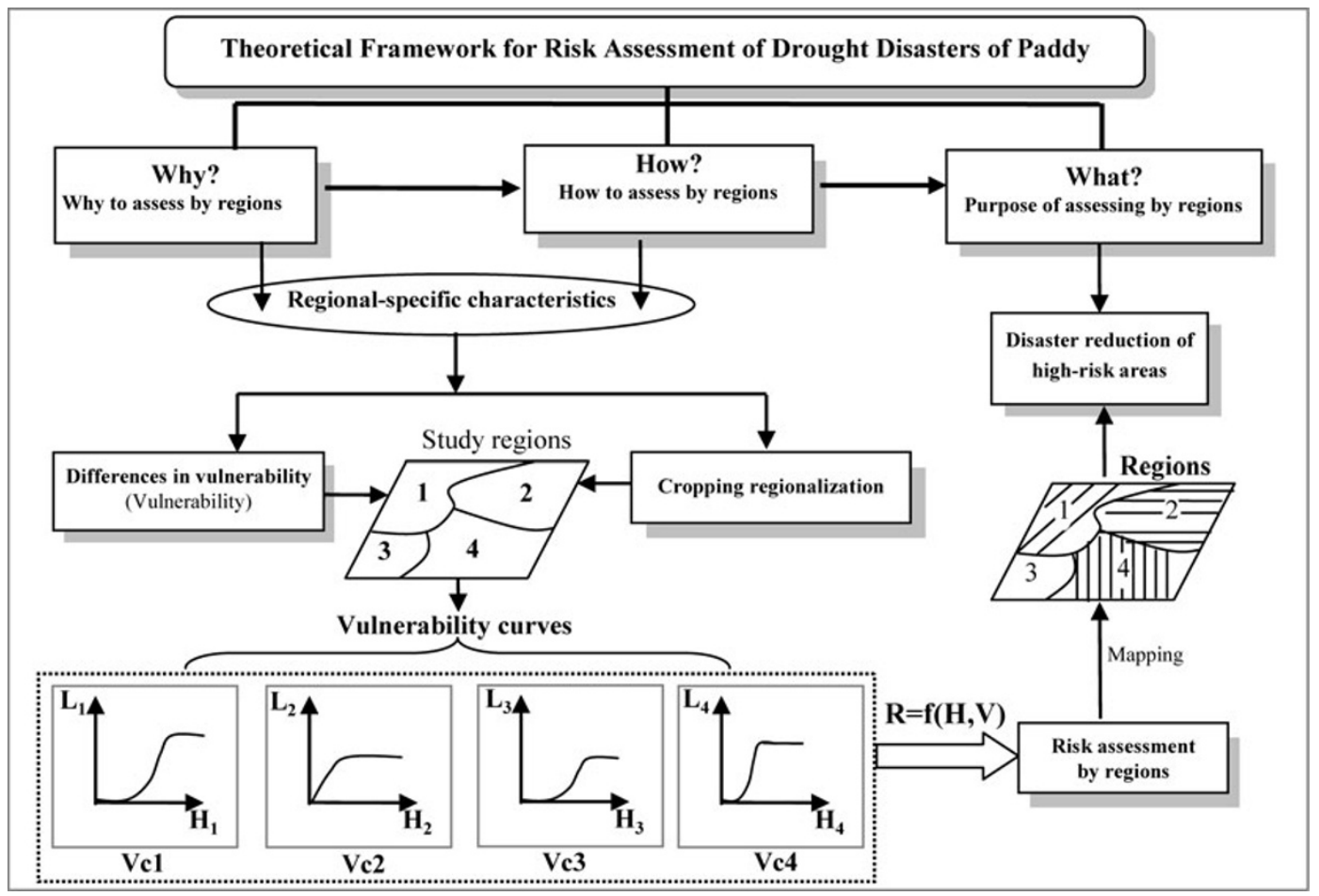

Figure 1. Framework for assessing the drought risk of paddy in China-the Regional Assessment-Vulnerability Variation (RAVV) framework (1, 2, 3, and 4 are area codes; $H$ represents hazard severity; V represents vulnerability to droughts; and $R$ is risk)

Table 2. National standard for the classification of drought hazards (by precipitation anomaly)

\begin{tabular}{llccc}
\hline \multirow{2}{*}{ Category } & & & Precipitation Anomaly (\%) \\
\cline { 3 - 4 } & & Mype & Quarterly & Annually \\
\hline 1 & Drought free & $-40<h$ & $-25<h$ & $-15<h$ \\
2 & Light drought & $-60<h<-40$ & $-50<h<-25$ & $-30<h<-15$ \\
3 & Medium drought & $-80<h<-60$ & $-70<h<-50$ & $-40<h<-30$ \\
4 & Severe drought & $-95<h<-80$ & $-80<h<-70$ & $-45<h<-40$ \\
5 & Exceptional drought & $h \leq-95$ & $h \leq-80$ & $h \leq-45$ \\
\hline
\end{tabular}

Source: China Meteorological Administration 2006.

Where, $P_{(h, t)}$ represents the exceeding probability of drought hazard at severity $h ; t$ represents the recurrence interval of certain drought hazard severity, $n$ represents the number of years of input data, from 1966-2005 (40 years, yearly total precipitation for the growing season).

\subsection{Establishing Regional Drought Vulnerability Curves for Mid-Season Paddy}

The establishment of vulnerability curves is the basis for the quantitative assessment of disaster vulnerability (Birkmann 2006, 2007). In order to conduct a regionally differentiated drought assessment, the Chinese mid-season paddy area is divided into four main regions: South China, North China, Northeast China, and Northwest China, while the QinghaiTibet Plateau is not considered because it is not suitable for growing paddy. Based on the cases of drought disaster losses, we establish the correlation between drought hazard severity (h) and drought disaster affected ratio (the ratio of the disaster affected area to the total mid-season paddy cropping area in a region), and use the non-linear statistical model in Matlab 7.1 for multiple fitting and accuracy testing and regression to obtain the regional vulnerability to drought curve of mid-season paddy (Eq. 3).

$$
Y_{(h)}=\frac{a}{\left(1+b \times e^{c \cdot h}\right)}+d
$$

Where, $Y_{(h)}$ represents the drought disaster affected ratio under certain drought hazard severity $h ; a, b, c$, and $d$ are model parameters. Vulnerability is usually portrayed as the susceptibility to be harmed (Adger 2006). In this article, regional drought vulnerability is defined as the potential 
disaster affected ratio of a region under certain drought stress. With the same drought hazard severity, the larger the drought disaster affected ratio, the higher the vulnerability of the region.

\subsection{Calculating the Drought Disaster Risk of Mid- Season Paddy}

The drought disaster risk of mid-season paddy for each $1 \mathrm{~km} \times$ $1 \mathrm{~km}$ grid is the product of the exceeding probability of drought hazard at severity $h$ and the drought disaster affected ratio under the corresponding hazard severity $h$ (Eq. 4).

$$
R=P_{(h, t)} \times Y_{(h)}
$$

Where, $R$ represents the drought disaster risk index; $P_{(h, t)}$ represents the exceeding probability of drought hazard at severity $h ; Y_{(h)}$ represents the drought disaster affected ratio under the corresponding drought hazard severity $h$.

\section{Results and Analysis}

Based on the quantitative risk assessment model for drought disaster of mid-season paddy, the severity of drought hazard, drought vulnerability, and the risk of drought disaster for mid-season paddy in China are assessed in this section.

\subsection{Severity Assessment of Drought Hazard for Mid- Season Paddy in China}

Figure $2 \mathrm{a}-\mathrm{d}$ shows the distribution of drought hazard severity for mid-season paddy (Eq. 1) at four drought recurrence intervals. With increasing recurrence intervals (from once in 5 years to once in 10,20, and 40 years), areas suffering from severe drought and exceptional drought in China increase, areas free of drought and suffering from only light drought decrease, and areas of medium drought first increase and then decrease.

At the 5-year recurrence interval, drought impact was basically below the light drought level (97.6 percent of the area was in a state of light drought or drought free), without any area of severe drought and exceptional drought. At the recurrence interval of once in 10 years, areas of exceptional drought and severe drought started to appear, but the proportion was extremely low. Areas of medium drought expanded to 33.8 percent and areas of light drought decreased to 58.7 percent, while the drought-free areas disappeared. Under droughts of this recurrence interval, mid-season paddy suffered from drought to various degrees, with medium level and more severe droughts mainly concentrated in Northwest China, North China, and the northern part of the Yangtze River Basin. Under droughts of 20-year recurrence interval, the areas at risk of drought at and above the medium level expanded significantly ( 85 percent); areas of medium drought increased to 54.1 percent, areas of severe drought and exceptional drought decreased to 19.6 and 12.6 percent, and areas of light drought decreased significantly to 13.8 percent. Areas of exceptional drought were mainly concentrated in Northwest China, the central-southern part of North China, and the northern part of the Yangtze River basin. Under droughts of once in 40 years, areas of exceptional drought in the northern part of the Yangtze River Basin spread significantly, the impact level in Southwest China and the southern part of Guangdong Province increased sharply and 67 percent of the mid-season paddy area was in the category of severe drought or exceptional drought and the drought area below medium level basically disappeared. Under droughts of this recurrence interval, mid-season paddy in China suffered from drought above the medium level and areas of exceptional drought accounted for 43.1 percent of the entire mid-season paddy areas.

The severity of drought hazard for mid-season paddy shows a significant difference between the south and the north (Figure 2a-d): drought severity in Northwest China, North China, and the Yangtze River Basin is relatively high, but relatively low in Northeast China and South China, while drought severity in the Sichuan Basin remains at a relatively low level as well. As the recurrence interval increases, the areas of exceptional drought and severe drought clearly expand southeastward.

Northwest China, North China, and the Yangtze River Basin are the high-severity regions of drought hazard for mid-season paddy. Especially in the Northwest and the northern region of the Yangtze River Basin, drought severity is extremely high at any recurrence interval and special attention needs to be paid to drought prevention. The Northwest is the region of high drought prevalence in China, with low annual precipitation and high spatial and temporal variability. The middle and lower reaches of the Yangtze River have relatively abundant precipitation, but with uneven temporal distribution, both seasonally and interannually. Additionally, due to the impact of the unstable monsoon climate, continuously high-temperature and low-rainfall weather occurs frequently during the critical June/July growing period for mid-season paddy, resulting in late summer drought and even combined summer and autumn drought that increases the drought severity of mid-season paddy in the region. The paddy areas in Northeast China and South China are the main mid-season paddy cropping areas in China, with much higher precipitation and more surface water resources as compared with Northwest China and North China, and thus have a lower risk of drought.

\subsection{Vulnerability Assessment of Mid-Season Paddy in China}

Based on Eq. 2, the mid-season paddy drought vulnerability curve function (Eq. 5-8) and vulnerability curve fitting graph (Figure 3a-d) are obtained for the four main regions. $Y_{(h)}$ represents the drought disaster affected ratio; $h$ is the drought hazard severity index. The vulnerability curve fitting values $\mathrm{R}^{2}$ for the four regions are $0.843,0.824,0.901$, and 0.819 , all have passed the significance test. 


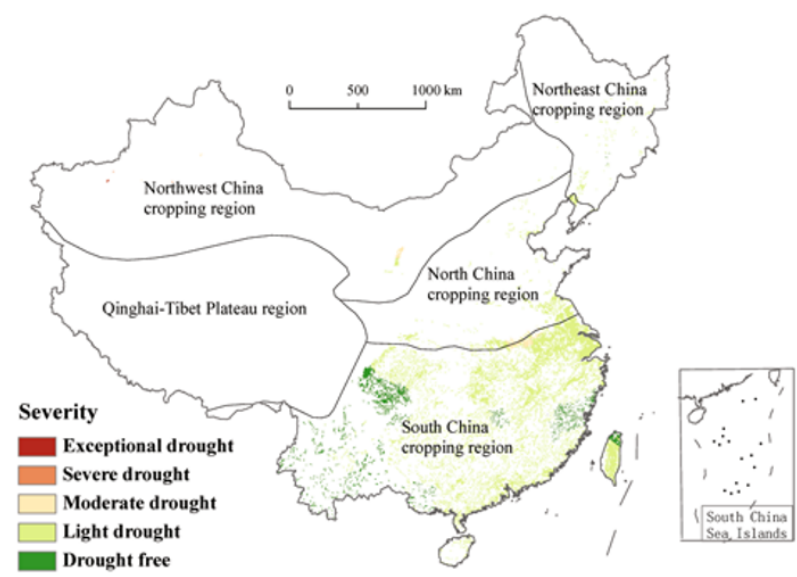

(a) Once in 5 years

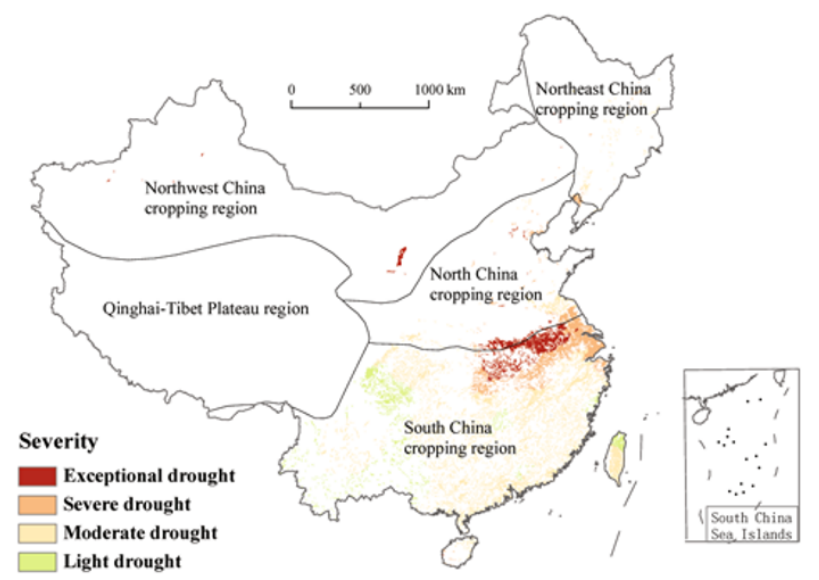

(c) Once in 20 years

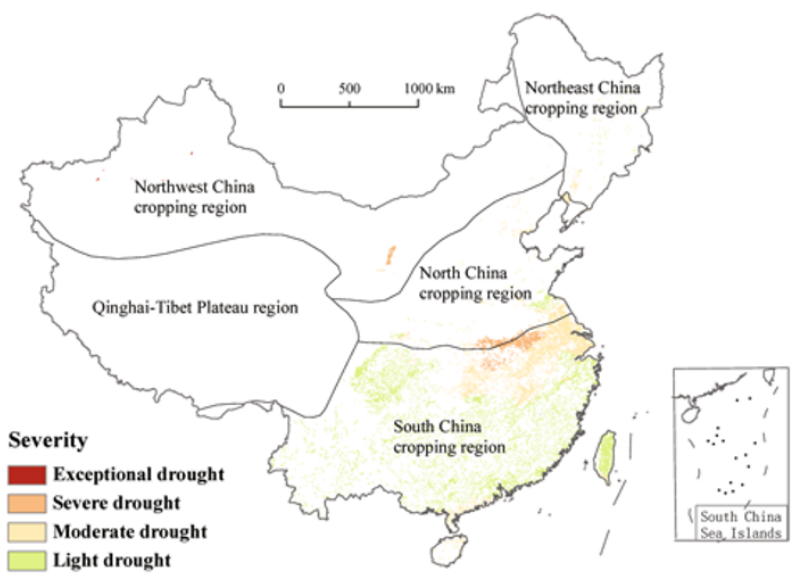

(b) Once in 10 years

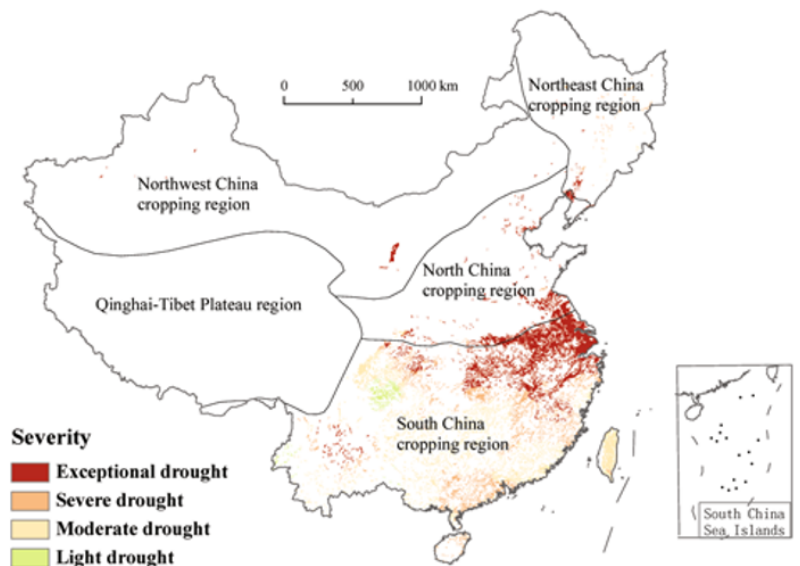

(d) Once in 40 years

Figure 2. Distribution of the drought hazard severity index for mid-season paddy at four drought recurrence intervals

South China: $\quad Y_{(h)}=0.355 /(1+6.490$

$\times \operatorname{Exp}((-4.765) \times h))+0.040$

Eq. 5

North China: $\quad Y_{(h)}=2.287 \mathrm{e}^{5} /\left(1+7.027 \mathrm{e}^{5}\right.$

$$
\times \operatorname{Exp}((-0.530) \times h))-0.140
$$

Eq. 6

Northeast China: $Y_{(h)}=0.544 /(1+56.892$

$$
\times \operatorname{Exp}((-6.752) \times h))+0.186
$$

Northwest China: $Y_{(h)}=0.407 /(1+9.942$

$$
\times \operatorname{Exp}((-5.524) \times h))+0.178
$$

By comparing the disaster impact at maximum hazard severity (Figure 3a-d), we can see that mid-season paddy vulnerability to drought is highest in Northeast China, followed by Northwest China and North China, while in South China vulnerability is lowest. Vulnerability in the north is higher than in the south. Due to the warming and drying trend in the north over the last five decades, some studies have indicated that the northeastern paddy area is a highly sensitive region impacted by climate change $(\mathrm{Fu}$ and $\mathrm{Ma}$ 2008). Weak and variable monsoon influence in the region also results in higher vulnerability to drought of mid-season paddy. The northwestern region has low annual precipitation and high seasonal and interannual variability in precipitation. Rapid industrialization and urbanization in the area compete with paddy cropping for water and lead to relatively high vulnerability of mid-season paddy in the region. Due to the impact of the monsoon climate, precipitation in North China is also erratic. However, since most paddy areas in the region have well-developed irrigation infrastructure, vulnerability to drought is relatively low here. South China is the region most suitable for growing mid-season paddy given its optimal light, temperature, and water resource conditions. At the same time, mid-season paddy vulnerability to drought is also the lowest compared with all other regions.

When drought hazard severity changes from 0.6 to 0.8 , the mid-season paddy vulnerability index increases sharply in all 


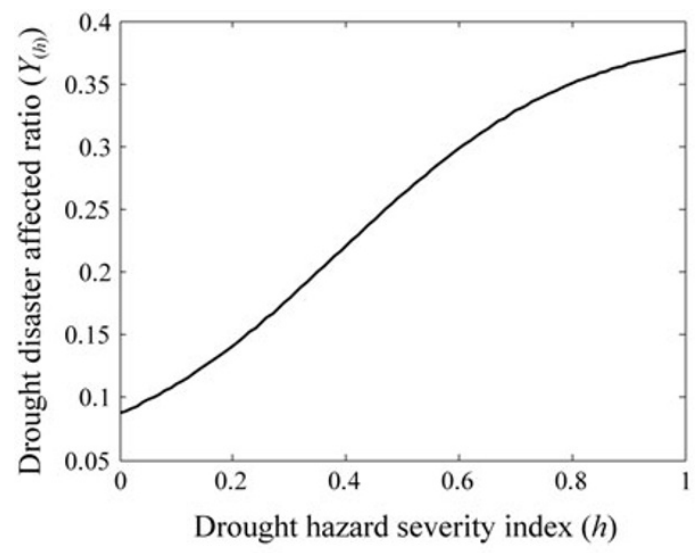

(a) Vulnerability curve of mid-season paddy in South China

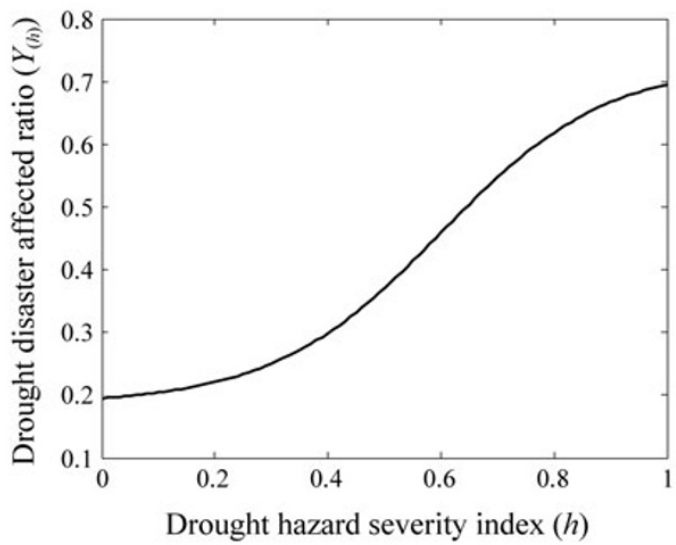

(c) Vulnerability curve of mid-season paddy in Northeast China

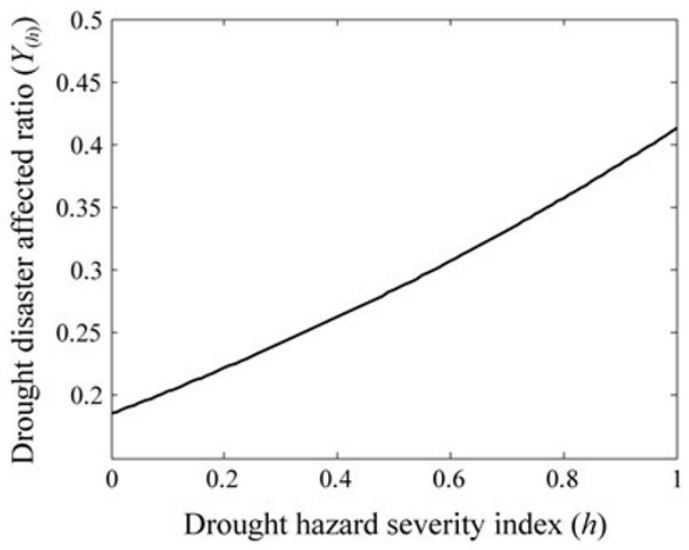

(b) Vulnerability curve of mid-season paddy in North China

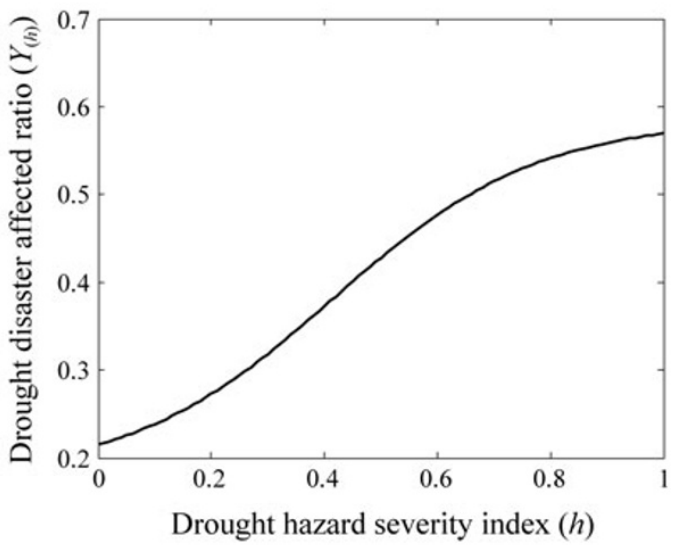

(d) Vulnerability curve of mid-season paddy in Northwest China

Figure 3. Vulnerability to drought of mid-season paddy in China

four regions and the rate of increase in Northeast China and Northwest China is higher than in North China and South China. Taking Northeast China as an example, when the hazard severity index increases from 0.6 to 0.8 , vulnerability changes from 0.2 to 0.6 . When drought severity is at medium and high levels, the drought impact is high and it becomes necessary to take preventive measures during this period in order to effectively reduce losses.

\subsection{Drought Risk Assessment of China's Mid-Season Paddy}

Based on the risk model of drought disaster (Eq. 4), the spatial distribution of mid-season paddy drought risk at 5-year, 10year, 20-year, and 40-year recurrence intervals (Figure 4) is obtained. For easy comparison, disaster risk is classified into five grades: a risk index of $0.1-0.3$ represents low risk; 0.3-0.4 represents relatively low risk; $0.4-0.5$ represents medium risk; $0.5-0.6$ represents relatively high risk; and a risk index above 0.6 represents high risk.
A comparison of the resulting graphs of the risk of drought disaster for mid-season paddy in China (Figure 4) shows a declining trend from Northeast China, Northwest China, North China, to South China, and the difference between the level of risk in the north and the south is very clear. Regional differentiation in climatic conditions, especially the difference between water and temperature conditions in the north and the south, is the main reason for the regional variation of drought disaster risk of mid-season paddy.

In view of the regional drought disaster system, there are mainly two reasons for this difference: (1) drought hazard severity is higher in the north than in the south; and (2) vulnerability of mid-season paddy is also higher in the Northeast and Northwest regions than in North China and South China. The regional difference in vulnerability has contributed greatly to the results of the risk assessment. For instance, the drought hazard severity of Northeast China is not particularly high, but the extremely high vulnerability of mid-season paddy results in the high drought disaster risk of mid-season paddy. It also illustrates that drought disaster risk of crops 


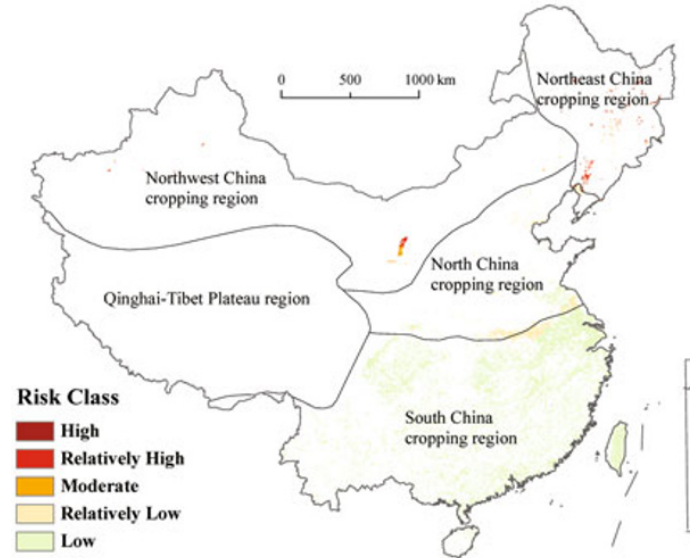

(a) Once in 5 years

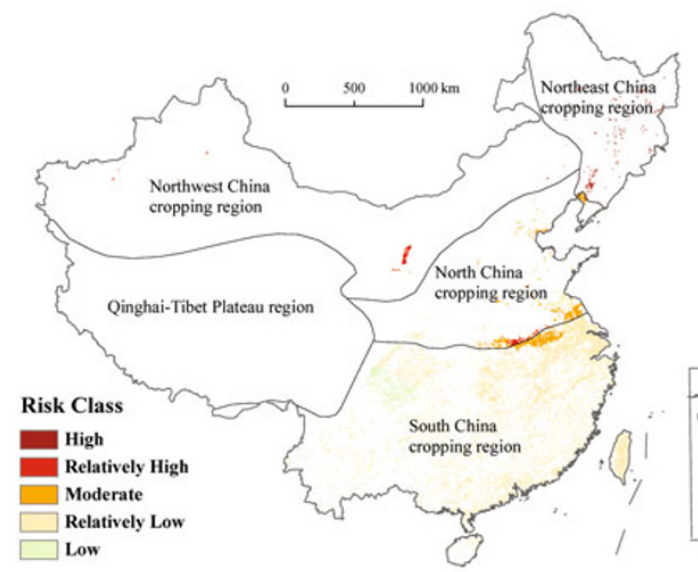

(c) Once in 20 years

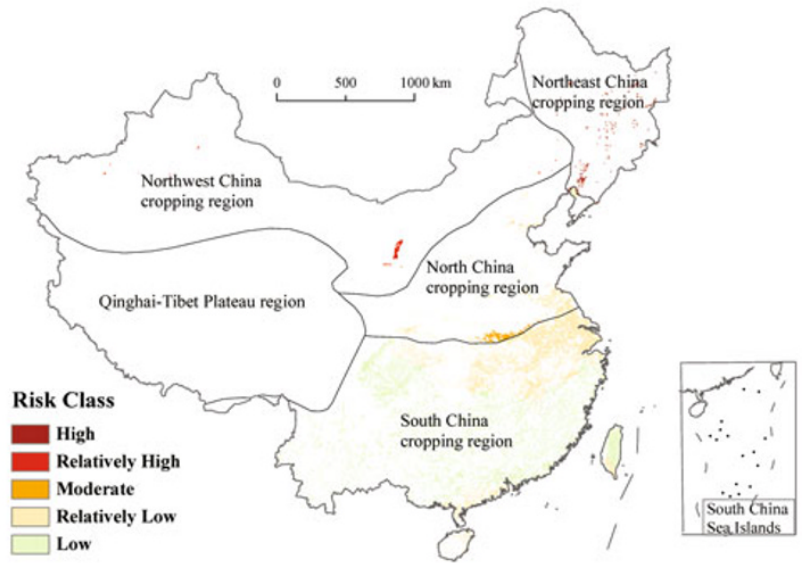

(b) Once in 10 years

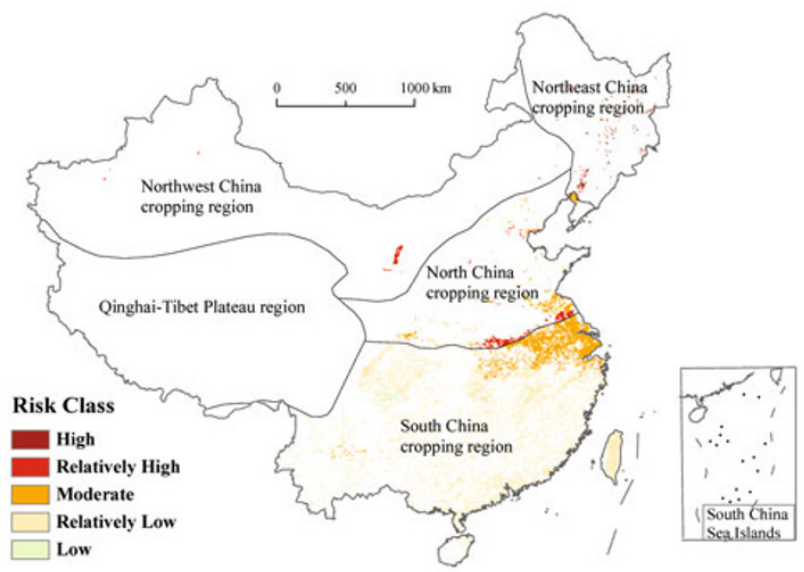

(d) Once in 40 years

Figure 4. Drought disaster risk of mid-season paddy in China

results from the combined effect of the hazard and the vulnerability of the exposure units (paddy crops), as well as the instability of the local environment.

By extracting statistics of disaster risk within each province in China, the mean and standard deviation of risk values of drought disaster of mid-season paddy within different provinces are derived. Since risk among provinces differs the most at the 10-year recurrence interval, our analysis is focused on comparing the mean value and standard deviation of risk for different provinces at the 10-year recurrence interval level. In view of the mean risk value (Figure 5), the Northeast region (Heilongjiang, Jilin, and Liaoning Provinces) has extremely high values (at $0.58-0.71$ ). For the Northwest region including Inner Mongolia, Xinjiang, and Ningxia, the risk value (at $0.5-0.6$ ) is lower than in the Northeast, while the risk value for the provinces in North China such as Beijing, Tianjin, Hebei, Shandong, and Shanxi is only $0.3-0.5$, lower than in the Northeast and Northwest as a whole, and the risk value of the vast southern provinces is even lower.

The standard deviation of risk value within each province reflects the spatial difference of risk within the province. Since Inner Mongolia has greatly varied natural conditions from the east to the west and is at the transitional zone between agriculture and animal husbandry with very high ecological vulnerability, mid-season paddy production in the province is very unstable and the spatial variation of drought disaster risk of mid-season paddy is the highest although its mean value is not particularly high. Difference in risk within Liaoning Province is also very high. In Sichuan, Chongqing, and Yunnan of the southwest, mean risk values are relatively low but standard deviation of risk values within each of these provinces is higher than most of the other southwest provinces. The southwestern region is rich in surface water resources, but with a complex topography and unstable climatic system, showing a high internal spatial difference of 


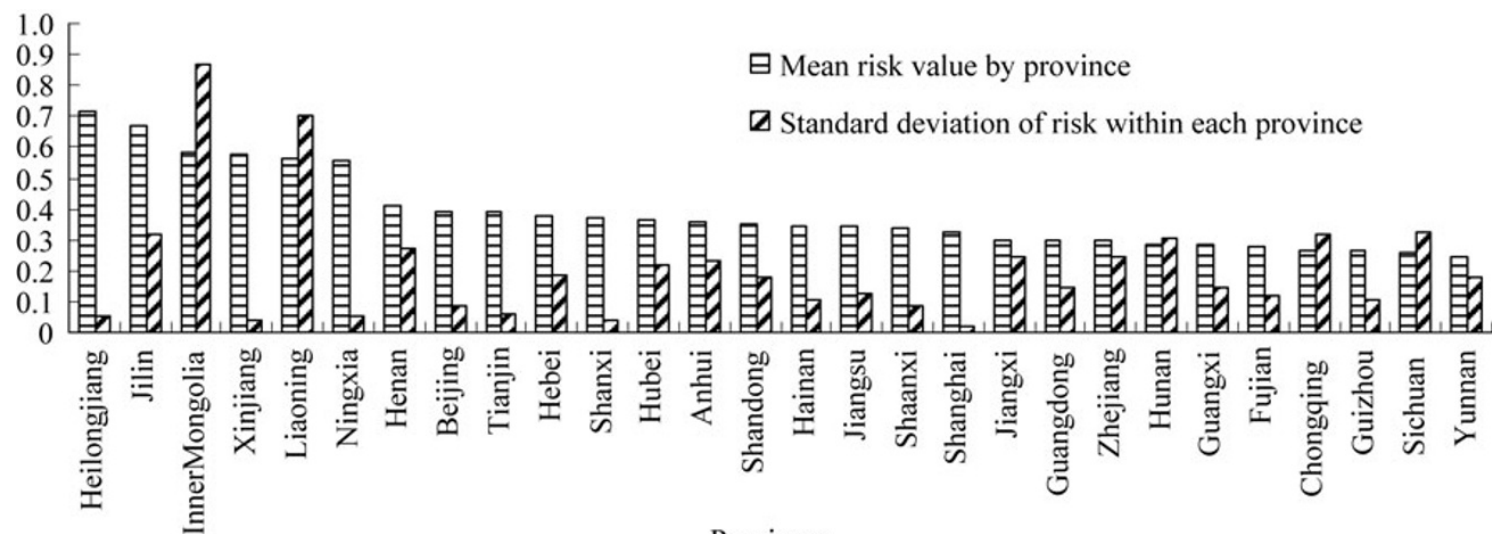

Provinces

Figure 5. Statistics of drought disaster risk value by province

drought risk. During the severe drought of the five southwestern provinces in the spring of 2010, the drought conditions were relatively severe for the region as a whole but differed greatly between provinces and areas of different topography.

\section{Conclusion}

Based on the theory of regional disaster systems and the regionally differentiated characteristics of paddy production, this article carried out a quantitative assessment of drought disaster risk for mid-season paddy in China. Based on the exceeding probability of drought hazard and vulnerability curves by regions, we evaluated for the first time the risk of drought disaster for mid-season paddy in China and compiled risk maps of drought disaster at four recurrence intervals, which can be a useful reference for the future study of agricultural drought disasters.

Northwest China, North China, and the Yangtze River Basin are the high-severity regions of drought hazard for mid-season paddy; the Northeast has the highest mid-season paddy vulnerability, followed by Northwest China and North China, while drought disaster risk is lowest in the south. By attaching special importance to the prevention of drought hazard of medium and high severity, the vulnerability of mid-season paddy can be significantly reduced. The risk of drought disaster for mid-season paddy in China shows a clear declining trend from Northeast China to Northwest China, North China, and South China, and the difference between the level of risk in the north and the south is significant. By quantitatively assessing risk by regions, this article offers a preliminary answer to where the high risk areas of drought disaster for mid-season paddy are and how high the risks are, and provides a regional-specific basis for risk prevention of drought disasters for paddy.

One of the deficiencies in this research is that due to the lack of highly accurate distribution data of mid-season paddy in China, paddy fields from a land-use map were taken as the areas of mid-season paddy cropping, which differ somewhat from the actual distribution range of mid-season paddy. Second, when establishing the regional vulnerability curves, due to limitations such as inadequate data of paddy drought loss cases, we assumed uniform distribution of rice variety and growing period in each region. In the future, based on more detailed data on variety, growing season, and corresponding drought losses within each region, the vulnerability curves can be refined to increase the spatial-temporal resolution of the vulnerability assessment.

In building the risk assessment model for mid-season paddy, this research has taken into account hazard severity, exposure, and vulnerability of the exposure units (paddy crops). In the future by including factors of the disaster formative environment such as topography and disaster coping capacities such as irrigation conditions in the risk assessment model, the accuracy of the results can be improved. Furthermore, the risk probability distribution calculated in this research was based on historical data from 1966-2005, without taking into consideration future climate change. As a next step, the risk assessment model can be matched with the General Circulation Model by scenarios to achieve an improved quantitative assessment of drought disaster risk for paddy under global climate change.

\section{Acknowledgments}

This study was supported by the National Natural Science Foundation of China (41171402) and the National Scientific and Technological Supporting Program of China (2008BAK50B07). Appreciation is extended to Professor Peijun Shi of Beijing Normal University for his guidance on the overall structure and content of this article.

\section{References}

Adger, W. N. 2006. Vulnerability. Global Environmental Change 16 (3): 268-81.

ADRC (Asian Disaster Reduction Center). 2005. Total Disaster Risk Management: Good Practice. Kobe, Japan: Asian Disaster Reduction Center. 
Birkmann, J., ed. 2006. Measuring Vulnerability to Natural Hazards: Towards Disaster Resilient Societies. Tokyo: United Nations University Press.

- 2007. Risk and Vulnerability Indicators at Different Scales: Applicability, Usefulness and Policy Implications. Environmental Hazards 7 (1): 20-31.

CAS (Chinese Academy of Sciences). 1989. China National Agricultural Atlas. Beijing: China Map Press (in Chinese).

Chen, X. H. 2009. China Agricultural Statistical Data. Beijing: China Agriculture Press (in Chinese).

China Meteorological Administration. 2006. Classification of Meteorological Drought Hazards (GBT20481-2006). Beijing: Standards Press of China (in Chinese).

Chinese Academy of Agricultural Sciences. 2010. Agridata. http://www. agridata.cn/ (in Chinese).

Department of Cropping Administration, Ministry of Agriculture. 2010. Grain. http://zzys.agri.gov.cn/zzy_cn.aspx?filenum=cereals (in Chinese).

Ding, Y. H. 2008. China Meteorological Disasters Collection. Beijing: China Meteorological Press (in Chinese).

Fu, Z. B., and Z. G. Ma. 2008. Global Change and Regional Aridification. Atmospheric Sciences 32 (4): 752-60 (in Chinese, English summary).

IGSNRR, CAS (Institute of Geographic Sciences and Natural Resources Research, Chinese Academy of Sciences). 2000. 1:1,000,000 China Land-Use Vector Format GIS Map.

IPCC (Intergovernmental Panel on Climate Change). 2007. Summary for Policymakers. In Climate Change 2007: Climate Change Impacts,
Adaptation and Vulnerability. Cambridge: Cambridge University Press.

National Bureau of Statistics of China. 2001. China County and City Socioeconomic Statistical Yearbook. Beijing: China Statistics Press (in Chinese).

NDRCC (National Disaster Reduction Center of China). 2008. Monthly Bulletin of Disaster Conditions. Beijing (in Chinese).

Shi, P. J. 2002. Third Discussion on Theory and Practice for Systematic Studies on Disasters. Journal of Natural Disasters 11 (3): 1-9 (in Chinese, English summary).

. 2005. Fourth Discussion on Theory and Practice for Systematic Studies on Disasters. Journal of Natural Disasters 14 (6): 1-7 (in Chinese, English summary).

. 2009. Fifth Discussion on Theory and Practice for Systematic Studies on Disasters. Journal of Natural Disasters 18 (5): 1-9 (in Chinese, English summary).

UNDP (United Nations Development Program). 2004. Reducing Disaster Risk: A Challenge for Development. Geneva: United Nations Development Program.

Wang, J. A., Y. X. Shang, Y. Su, Z. Q. Wang, and C. Y. Yang. 2005. Vulnerability Diagnosis of Disaster-Affected Body of Agricultural Drought in China and Regional Sustainable Development. Journal of Beijing Normal University (Social Sciences) 2005 (3): 130-37 (in Chinese, English summary).

Wang, J. A., H. Sun, W. Xu, and J. J. Zhou. 2002. The Temporal-Spatial Changes of Drought Disasters in China over the Last 5 Decades. Journal of Natural Disasters 11 (2): 1-6 (in Chinese, English summary).

Open Access This article is distributed under the terms of the Creative Commons Attribution License which permits any use, distribution, and reproduction in any medium, provided the original author(s) and source are credited. 